\title{
Helicopter Control Using Fuzzy Logic and Narma-L2 Techniques
}

\author{
Noor Salam Al-Fallooji \\ Department of Electronic and Computer Engineering, School of Engineering, Design and Physical Science, Brunel \\ University London, UK \\ E-mail: 1442218@alumni.brunel.ac.uk

\section{Maysam Abbod} \\ Department of Electronic and Computer Engineering, School of Engineering, Design and Physical Science, Brunel \\ University London, UK \\ E-mail: maysam.abbod@brunel.ac.uk
}

Received: 06 June 2019; Accepted: 07 September 2019; Published: 08 October 2020

\begin{abstract}
Helicopter instability represents a key issue in non-linear applications that should be addressed. Accordingly, researchers are invited to design a robust and reliable controller to obtain a stable system and enhance its overall performance. The present study focuses on the use of the intelligent system in controlling the pitch and yaw angles. This lead to controlling the elevation and the direction of the helicopter. Further to the application of the Linear Quadratic Regulator (LQR) controller, this research implemented the Proportional Integral Derivative (PID), Fuzzy Logic Control (FLC), and Artificial Neural Network (ANN). The results show that FLC achieved a good controllability for both angles, particularly for the pitch angle in comparison to the nonlinear auto regressive moving average (NARMA-L2). Moreover, NARMA-L2 requires further improvement by using, for example, the swarm optimization method to provide better controllability. The PID controller, on the other hand, had a greater capability in controlling the yaw angle in comparison to the other controllers implemented. Accordingly, it is suggested that the integration of PID and FLC may lead to more optimal outcomes.
\end{abstract}

Index Terms: Fuzzy logic controller (FLC), Nonlinear systems, Helicopters, NARMA-L2.

\section{Introduction}

The widespread use of helicopters in short distance travelling is a result of their capability to land from and take off in small areas [1]. The helicopter is a nonlinear, complex, and unstable system that needs a reliable control to achieve safe and efficient flight. Helicopters are used worldwide in different sectors such as medical, military, civil, and police operations. The great demands of the small size lead to its use in many services, for example, traffic control, rescue, fire fighting tourism, and air-sea [2,3]. It has a heavy cross-coupling that occurs between six degrees of freedom which are up-down, right-left, forth-back, roll, pitch, and yaw [2]. It flies in different directions and in three-spatial dimensions enclosing vertical, lateral, and longitudinal manoeuvres [1]. Such features, however, mean that its control is a challenging process [1,4]. Moreover, a simple movement may need more than one instruction at time to control it. Hence, pilots need a significant amount of skills and experience to fly the helicopter safely. It has a monitoring system with a three command bar. These commands are provided for a pilot for controlling purposes [2]. The first command is cyclic which is responsible for changing the pitch of the main rotor blades and directing the helicopter in the desired direction. The second command is the collective pitch which is used to control the amount of lift force generated by the main rotor and also controlling the angle of pitch. The third command is the pedal tail, which is responsible for providing torque to prevent the helicopter from spinning around itself [2,5]. However, the aforementioned commands and pilot experience alone are not enough to provide a high level of controllability and stability for the helicopter.

Accordingly, an additional control is needed to provide safe and efficient aviation. To this end, intelligent system methods including fuzzy logic control (FLC) and artificial neural network (ANN) can be considered as one of the optimal solutions for achieving this goal. This is due to their capability for controlling nonlinear systems [6,7]. In this research, the Quanser two degrees of freedom helicopter (2-DOF) model was chosen, providing the rotation around the pitch and yaw axis. The model control uses feed forward, linear quadratic regulator, and integral control $(\mathrm{FF}+\mathrm{LQR}+\mathrm{I})$.

This research adds many significant contributions in comparison to earlier work. First, it extends previous 
literature on controlling non-linear systems. Moreover, the paper enhances the controllability and instability of the helicopter by designing different controllers using intelligent system techniques. Finally, it highlights the most suitable control that can be used in such non-linear applications. These results can be applied for real life applications.

The rest of this paper is structured as follows. Section two reviews earlier literature on the use of controllers in nonlinear applications, particularly helicopter systems. This is followed by explaining the mathematical model of the two degree of freedom helicopter along with the design of three controllers for this model. Section three also shows the implementation of the original control. The simulation results of the pitch and yaw angels are presented and discussed in Section four. Finally, the last Section concludes the main points presented in this research.

\section{Related Work}

Basri, Husain, \& Danapalasingam [8] designed a MIMO (multi-input-multi-output) controller for a quadrotor helicopter by implementing an intelligent adaptive back stepping with unknown source of noise. As an approximation disorder, a radial basis function neural network (NN) was used. Through the learning process, the parameters were changed. The research also used the fuzzy compensator method to reduce the amount of error that might be generated by the neural approximation. A theoretical enhancement in the helicopter system was approved. However, the study failed to provide an empirical implementation to confirm its findings.

Hernandez-Gonzalez, Alanis, Hernandez-Vargas [1] discussed some of the main challenges in the helicopter system such as the nonlinearity and the robust inter-couplings. In order to tackle these issues, the researchers used high order neural network (HONN) technique because of its importance to approximate the nonlinearity in the Quanser 2 degree of freedom (2-DOF) helicopter model. With a view to enhancing the controllability of the yaw and pitch positions, the study adopted two techniques which are the neural back stepping and neural sliding mode block control. The whole system was divided into two subsystems by using the first method and these two subsystems, in turn, tracked the pitch and yaw positions. The second technique (block control) was implemented to HONNs. It was used to identify the helicopter system model. In each discrete-time, an online training with an extended Kalman filter based technique was used in the neural network training. An excellent performance of both techniques adopted was shown and without prior knowledge of neither the plant parameters nor its model [1]. However, it was found that the second method achieved slightly better results in comparison to the back stepping one. This improvement was attributed to the receiver of further information by the block control.

In order to enhance the control of a novel Gyroscopic Inverted Pendulum (GIP) device, Non-Linear Auto Regressive Moving Average (NARMA-L2) control technique was adopted [9]. The GIP plant includes a controller which is known as the inverted pendulum new class (IPNC). The IPNC encompasses the PID controller. In the first stage, the NARMA-L2 method was used to IPNC controller by determining the plant model. The second stage trained the neural network in order to represent the system's forward dynamic. The Simulink model included both the design of the NARMA-L2 as well as the PID controller. The use of the available switch block between the controllers allowed for the shifting from one controller to another. Based on the implementation of both methods, the outcomes indicated that the shift from the PID to the other controller (NARMA) could produce increased controllability. Moreover, the steady state error was reduced and the oscillation of the output signal was less. It was suggested that the use of NARMA model can improve the control more than the PID model.

In another research study [10], a fuzzy PID (FPID) control was developed based on adjusting the three parameters of the PID control ( Kp, KI, KD) using fuzzy rules. The adjusted FPID control was used to stabilize the nonlinear quad rotor helicopter model. The FPID control was converted into a real time system using dSPACE platform to produce a control signal for quad rotor helicopter. The proposed method had successfully reduced the overshoot of the classical PID.

In [11], a combination of adaptive and quantitative feedback theory (QFT) was used to control the 3-degree of freedom helicopter model. The results clearly showed an enhancement in the performance of the elevation, pith, and travel angles by applying the new controller. In addition, the overshoot and steady state error were reduced with adaptive and QFT controller in comparison to the original Linear Quadratic Control (LQR).

Singh and Panjwani [7] compared the effectiveness of fuzzy logic and NARMA-L2 controllers to control two links of a rigid manipulator. This application mimics the human upper arm which is known as a robotic arm. It is a nonlinear and complex system that needs special consideration in its design. As such, the study suggested using FL and NARMAL2 because of their ability to deal with the nonlinearity and parametric uncertainty. Another reason that led to choosing NARMA-L2 is its capability in controlling the path of such nonlinear system by eliminating dynamic behaviour [7]. The overall findings suggested that FL controller had the ability to achieve better outcomes than the NARMA-L2 controller. The settling time and peak overshoot were reduced with the former method (FL) by $70 \%$ and $60 \%$ for link1. On the other hand, the settling time decreased by $60 \%$, whereas the peak overshoot was increased by $44 \%$ for link 2 with FL controller in comparison to the NARMA-L2. 


\section{Methods}

\subsection{Mathematical model of the Quanser two degrees of freedom (2-DOF)}

The Quanser 2-DOF helicopter model was chosen in this study because of its flexibility in controlling the pitch and yaw angles (see Fig. 1). In this model, researchers can design a new controller based on their needs to control nonlinear applications. Moreover, the model can be applied for dynamic applications that are used in the real life. The model contains a helicopter placed on a fixed base together with two propellers. Each propeller is driven by a direct current (DC) motor. The elevation of the helicopter nose is controlled about the pitch axis by the front propeller, whereas the back propeller is responsible for controlling the side to side motion around the yaw axis [12]. The high-resolution encoders measure both pitch and yaw angles. The slipring, on the other hand, is used to transmit both pitch encoder and motor signals. The aim of using this form of transmitting is to prevent any tangling of the wires on the yaw axis and permits free rotation about 360 degrees for the yaw angle [13].

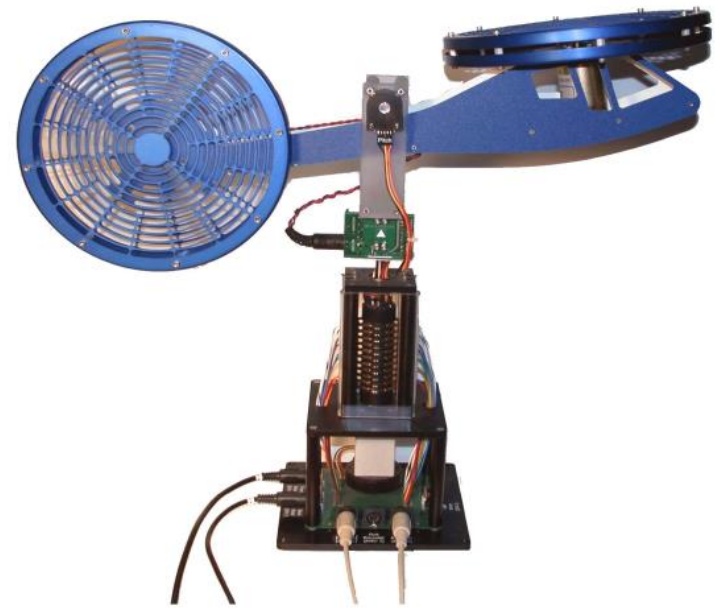

Fig.1. Quanser 2-DOF helicopter model [13]

Fig. 2 illustrates the free-body diagram of this model. It depicts the yaw and pitch axis where the pitch angle is $\theta$ and the yaw angle is $\psi$.

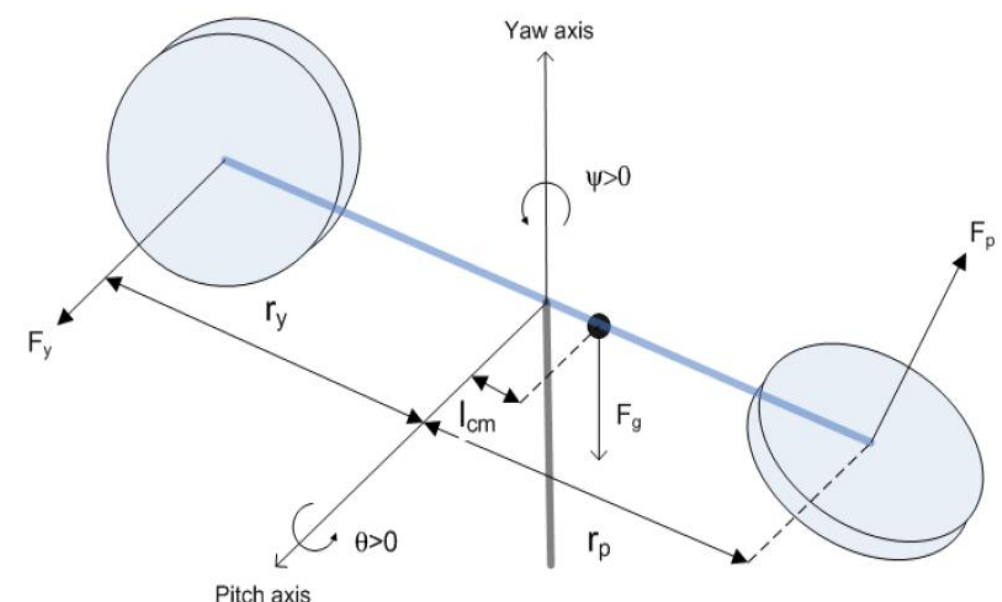

Fig.2. Free-body of 2-DOF the helicopter [13]

- In a case of the pitch angle is $\theta=0$, the helicopter will be horizontal.

- The pitch angle rises to more than zero $\theta(\mathrm{t})>0$ when the movement of the nose is up, and the rotation of the body is counter-clockwise $(\mathrm{CCW})$ direction.

- The yaw angle rises to more than zero $\psi(\mathrm{t})>0$ when the rotation of the body is clockwise $(\mathrm{CW})$ direction.

- Both pitch and yaw are positively rising to more than zero when the thrust force of the pitch is Fp $>0$ and the thrust force of the yaw is Fy $>0$. 
The thrust forces that are produced from the front and the back motors are represented on the axis of the yaw and the pitch. Moreover, the derivations of the nonlinear equations that represent the motion of the 2-DOF helicopter system are found by using Euler-Lagrange formula. Subsequently, the state space model (A, B, C, D) that expresses the joint of the voltage-angular is found as shown in the equations below, whereas the representation of the state space is given in (1) $[13]$.

$$
x=A x+B u, \text { and } y=C x+D u
$$

The state vector that consists of the pitch, yaw, the derivative of the pitch, and the derivative of the yaw angle is defined in (2). Furthermore, the output vector is defined in (3).

$$
\begin{gathered}
x^{T}=\left[\theta(t), \varphi(t), \theta^{\circ}(t), \varphi^{\circ}(t)\right] \\
y^{T}=[\theta(t), \varphi(t)]
\end{gathered}
$$

The matrices of the state space of the helicopter system are shown in (4, 5, 6, and7) [12][13].

$$
\begin{gathered}
A=\left[\begin{array}{rrrr}
0 & 0 & 1 & 0 \\
0 & 0 & 0 & 1 \\
0 & 0 & -\frac{B_{P}}{J_{T P}} & 0 \\
0 & 0 & 0 & -\frac{B_{y}}{J_{T y}}
\end{array}\right] \\
B=\left[\begin{array}{cc}
0 & 0 \\
0 & 0 \\
\frac{K_{P P}}{J_{T P}} & \frac{K_{p y}}{J_{T P}} \\
\frac{K_{y p}}{J_{T P}} & \frac{K_{y y}}{J_{T y}}
\end{array}\right]
\end{gathered}
$$

where $J_{T P}=J_{e q-p}+m_{\text {heli }} I^{2} c m \quad J_{T y}=J_{e q-y}+m_{\text {heli }} I^{2} c m$

$$
\begin{gathered}
C=\left[\begin{array}{llll}
1 & 0 & 0 & 0 \\
0 & 1 & 0 & 0
\end{array}\right] \\
D=\left[\begin{array}{ll}
0 & 0 \\
0 & 0
\end{array}\right]
\end{gathered}
$$

\subsection{Controlling Design}

Three types of controllers were designed to fix the limitation of the original model's control (Feed forward + Linear Quadratic Regulator+ Integral). These controllers are:

(1) Feed forward (FF)+ Linear Quadratic Regulator (LQR)+ Integral (I) Control: This represents the original control of the model. The FF+LQR+I controller was developed in [12]. This controller is a state-feedback that is used to direct the 2-DOF helicopter in the required position by regulating both travel and elevation angles. The FF+LQR+I controller was applied with the nonlinear 2-DOF helicopter model as shown in Fig. 3. The desired angles were set at $20^{\circ}$ for both pitch and yaw angels at $0.0125 \mathrm{~Hz}$ frequency. In this present paper, the output of this controller was compared with the designed controller. 


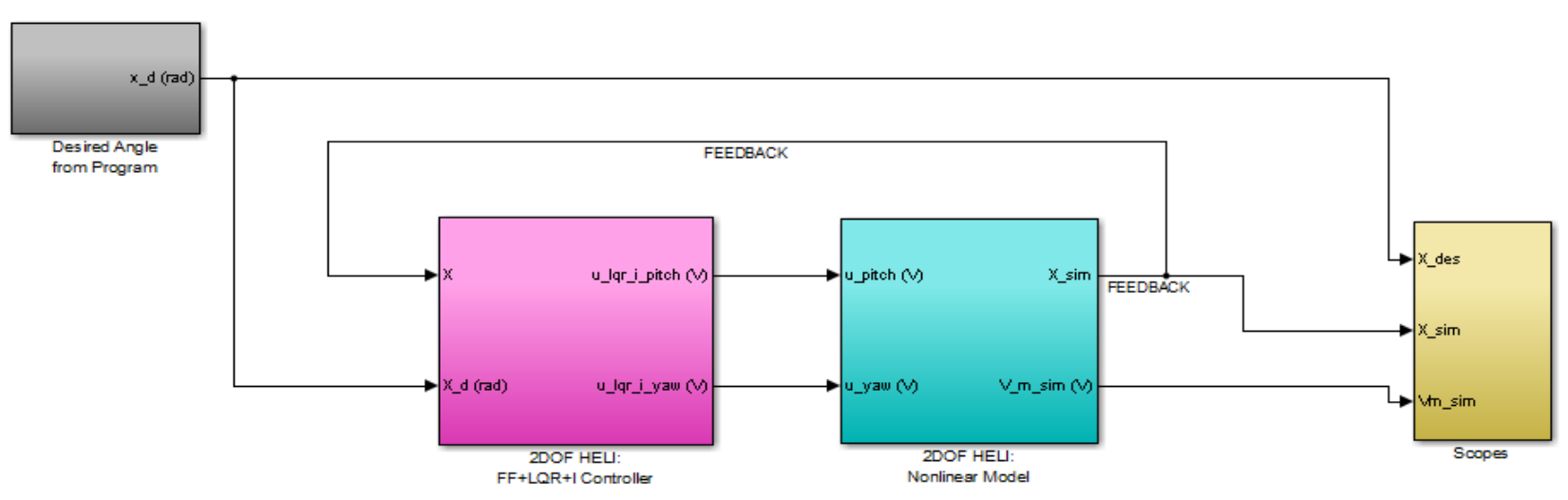

Fig.3. The Quanser 2-DOF model with FF+LQR+I control, closed-loop simulation

(2) PID: There are three types of this controller which are PI, PD and PID. The PID was chosen in this research based on its features in controlling nonlinear systems that require high reliability, good stability, and low toleration of offset error. The PID controller consists of three terms: proportional (P), integral (I), and derivative (D). Its main equation is illustrated in (8) [14].

$$
U(s) / E(s)=P+I / s+D s
$$

The PID control was implemented by replacing the original control of the model. For each angle (pitch and yaw), one controller was used as depicted in Fig. 4. The desired angles are square input in radian and the frequency value is $0.0125 \mathrm{~Hz}$. These angles were set at $20^{\circ}$, converted into radian, and separated for the pitch and yaw angles. Then, the output of the pitch and yaw angles were taken after converting it from radian into degree. The parameters of the PID were tuned for both controllers to reduce the steady state error and enhance its performance. The tuning process is a filter that was added to the controller to decrease the distortion, overshoot, and oscillation from the output signal as well as improving it to be closer to the desired output. The formula of the PID with the filter is exhibited in (9).

$$
\frac{U(s)}{E(s)}=P+\frac{I}{s}+D \frac{N}{N+1 / s}
$$

where $N$ is the filter coefficient. The parameters of the PID after tuning are:

$\mathrm{P}=90.0175453979879$

$\mathrm{I}=18.8837141818186$

$\mathrm{D}=35.9192179550268$

$\mathrm{N}=56.0907149512974$

(3) Fuzzy Logic Control (FLC): Another controller implemented in this research is the FLC. It has the ability of controlling nonlinear and imprecise systems. Moreover, this controller has strong interference and it is simple to be used in the design process [15].

The Multi-input multi-output (MIMO) FLC was designed as follows. Two FL were used to design the MIMO control for both pitch and yaw angles. Fig. 5 illustrates the FLC system that connected to the model. It contains two FL (FL1 and FL2) and each one has one input and one output.

The design of FLC like proportional derivative (PD) was done for each FL (Fig. 5). The first input in each FL consists of two components. The first one represents the $\mathrm{P}$ term which is the error, while the second input refers to the $\mathrm{D}$ term which is the change in error (a derivative of error). In addition, each input and output of the FL were provided with a gain to scale their parameters. The gain is crucial to enhancing the outcomes in which the Mamdani type was chosen. The value of the angle represents the output. Moreover, three membership functions for each variable were chosen. These functions are negative, zero, and positive respectively in the range $[-1,1]$. The type of the membership function is Gaussian. Based on (10) in which nine rules were chosen using Mamdani method.

$$
N=V^{m}=2^{3}=9
$$

$\mathrm{N}$ : number of rules.

$\mathrm{V}$ : number of variables.

M: the number of memberships function.

The nine rules were written as illustrated in Table 1. 


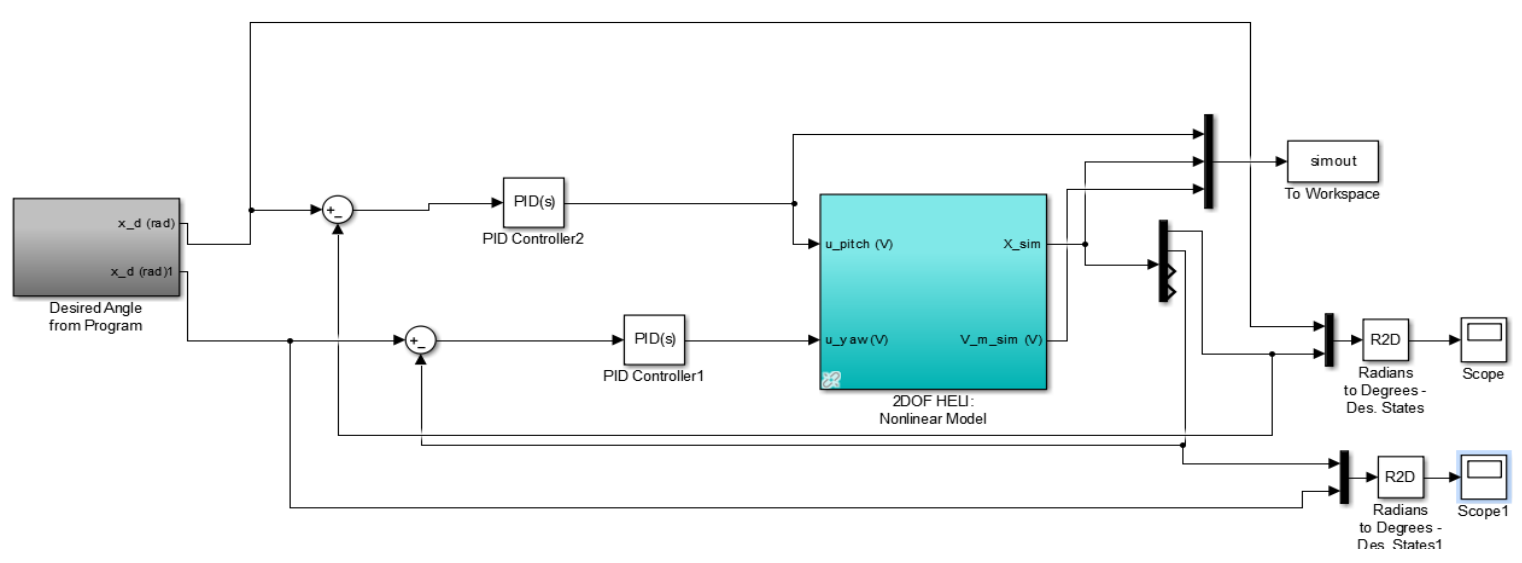

Fig.4. The design of PID control system for both pitch and yaw angles

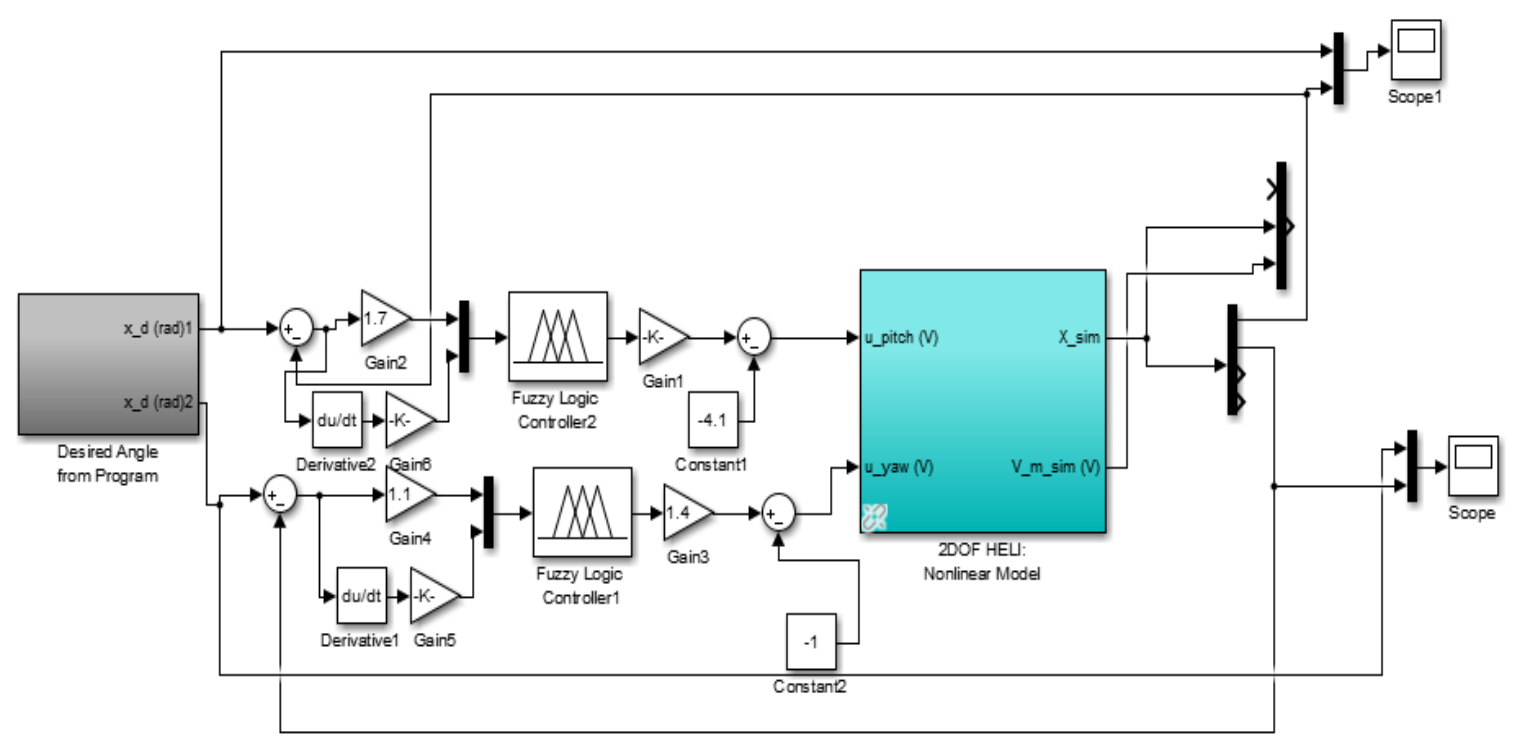

Fig.5. The FLC connected to the 2-DOF model

Table 1. The rules

\begin{tabular}{|c|l|}
\hline Number & Rules \\
\hline 1 & If (error is negative) and (change in error is negative) then (angle is negative) \\
\hline 2 & If (error is zero) and (change in error is negative) then (angle is negative) \\
\hline 3 & If (error is positive) and (change in error is negative) then (angle is zero) \\
\hline 4 & If (error is negative) and (change in error is zero) then (angle is negative) \\
\hline 5 & If (error is zero) and (change in error is zero) then (angle is zero) \\
\hline 6 & If (error is positive) and (change in error is zero) then (angle is positive) \\
\hline 7 & If (error is negative) and (change in error is positive) then (angle is zero) \\
\hline 8 & If (error is zero) and (change in error is positive) then (angle is positive) \\
\hline 9 & If (error is positive) and (change in error is positive) then (angle is positive) \\
\hline
\end{tabular}

The aforementioned steps were repeated to design the FLC for the yaw angle. Another step was implemented because of the nonlinearity behaviour of the yaw angle in comparison to the pitch angle. The adaptive neuro-fuzzy inference system (ANFIS) was used to tune the parameters of the FLC of the yaw angle and improve its performance. ANFIS has the advantages of both FL and NN methods. It uses back propagation algorithm to train the data online and adjust the parameters of the FL [16]. In this present research, ANFIS used 2 inputs and 1 output.

The input is the error of the yaw as well as the derivative of this error, whereas the output is the yaw angle value. The selected ANFIS is [-3,3]. This means three membership functions for each input and three membership functions for the output. The types of memberships are Gaussian and linear where the number of epochs is 20. The data was created using ANFIS designer and the system was trained as shown in Fig .6. Moreover, nine rules of the ANFIS were chosen automatically by using Sugeno method (auto method) as shown in Fig. 7. 


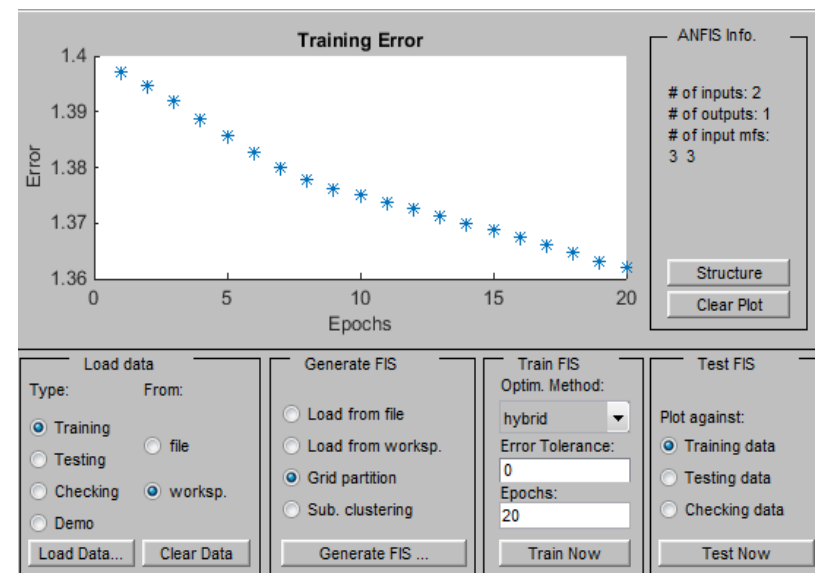

Fig.6. Training error in the ANFIS viewer

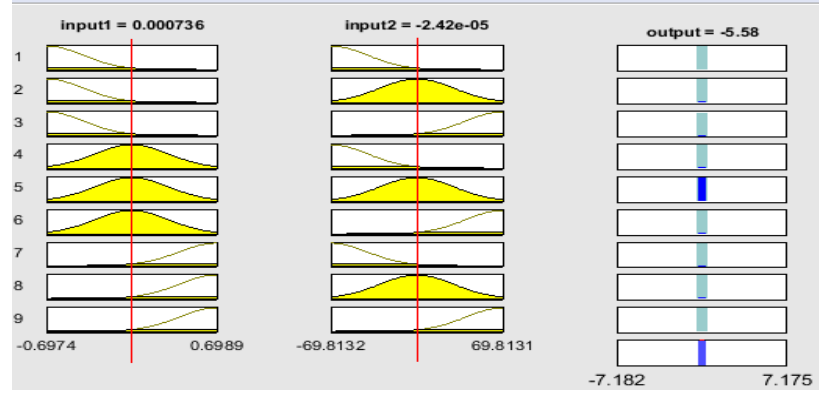

Fig.7. The rule base of the ANFIS viewer

The neural network of ANFIS trained the data in which the new FL was created after adapting ANFIS. It was exported to the fuzzy file in the form of FIS file. Then, the parameters of the yaw FLC was changed by the adapted parameters. This led to reducing the error between the output and the reference as depicted in Fig. 6.

(4) NARMA-L2 Control Based on Neural Network: The key concept of this control underlies the transformation of a non-linear system into linear dynamics based on excluding nonlinearities [17]. Two stages were followed to design this controller which are the system identification and control design. In the former, the design of an NN model of the plant is performed [18], whereas in the second stage, the identified plant model is used.

The reorganization of the plant model represents the design of the controller in NARMA-L2. It is trained offline and in batch structure [18]. This method uses less computation than other controllers such as model reference and model predictive. The use of NN as a controller requires adjusting the NARMA-L2 parameters and, thus, online control can be achieved. Particularly, the approximated methods are the only methods used in controlling the represented plant by the NARMA-L2 control. In the NARMA-L2 model, the first stage of the control is the identification. The widely used standard model for the nonlinear identification is called the nonlinear auto regressive-moving average (NARMA) model [17]. Equation 11 refers to the NARMA model of the discrete-time nonlinear system [9].

The approximation of (11) is the NARMA-L2 model. Hence, this model is computed by (12).

Fig. 8 presents the NARMA-L2 controller block diagram. To implement this controller, the aforementioned NARMA-L2 plant model can be used.

$$
Y(k+d)=[F(y(k), y(k-1) \ldots . . y(k-n+1)), u(k), u(k-1), \ldots . u(k-m+1)]
$$

where:

$\mathrm{u}(\mathrm{k})$ : the system input

$\mathrm{y}(\mathrm{k})$ : the system output, and

$\mathrm{d}$ : the system delay

$$
\begin{aligned}
& Y^{\sim}(k+d)=f[y(k), y(k-1), \ldots ., y(k-n+1), u(k-1), \ldots ., u(k-m+1] \\
& \quad+g[y(k), y(k-1), \ldots, y(k-n+1), u(k-1), \ldots, u 9 k-m+1)] . u(k)
\end{aligned}
$$

The following form represents the resulting controller: 


$$
u(k)=\frac{\operatorname{Yr}(k+d)-f[y(k), y(k-1), . ., y(k-n+1), u(k-1), \ldots, u(k-m+1)]}{g[y(k), y(k-1), . ., y(k-n+1), u(k-1), . ., u(k-m+1)]}
$$

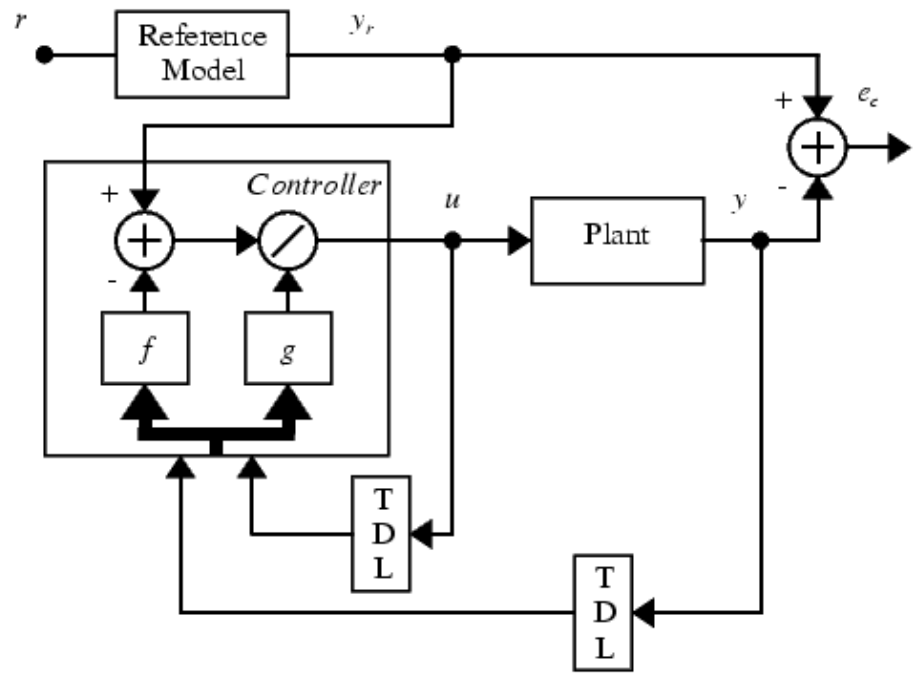

Fig.8. The controller of NARMA-L2 [9]

NARMA-L2 is used in the present study in order to control the 2-DOF helicopter model because of its capability of controlling uncertain and nonlinear systems [19]. This method uses the neural network to train the data. This can reduce the error and improve the output signal. This controller was designed in this research as follows:

- The identification of the system is an important factor in this method; therefore, the first step was generating the random input and output data by using the nonlinear model.

- The model was simulated and the input-output data was exported to the workspace.

- The NARMA-L2 controller of the pitch angle was designed by assigning zero to the yaw.

- The 2DOF model was run with NARMA-L2 where the generated data as shown in Fig. 9 was changed many times to achieve the desired stability. Based on this step, the simulation results were obtained.

The next stage was the design of NARMA-12 by assigning zero to the pitch angle in order to control the yaw angle.
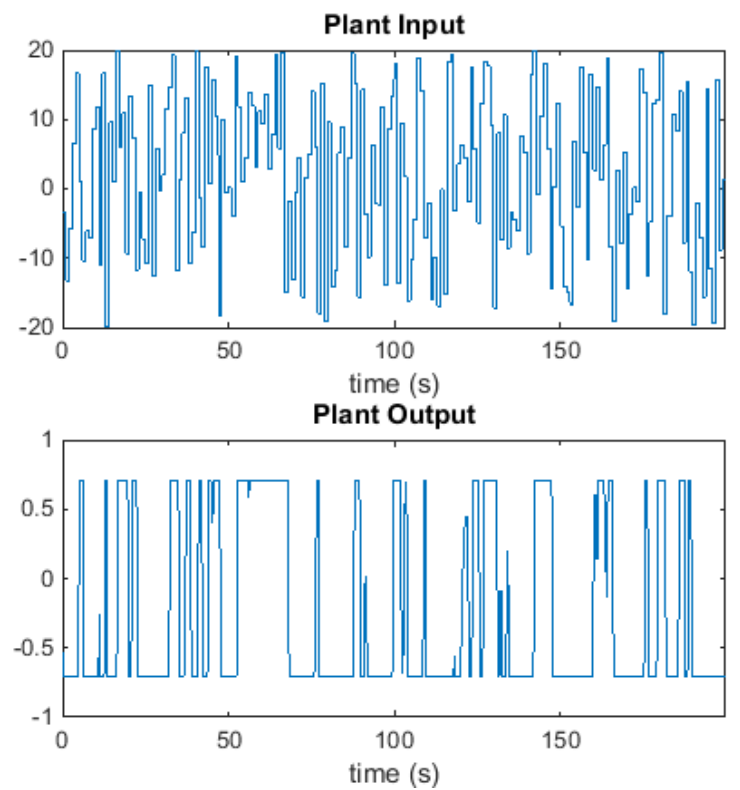

Fig.9. The pitch data generated and imported to the NARMA-L2

The steps were repeated for the yaw angle. While the range of the plant input data was [-15 15], the hidden layers were 10. Finally, both NARMA-L2 controllers were applied to the model in which the simulation results are obtained. 


\section{Results and Discussion}

The simulation results for the pitch and yaw angles of the FF+LQR+I controller are shown in Fig. 10 and Fig. 11 respectively. The $\mathrm{x}$-axis refers to the time in seconds, while the $\mathrm{y}$-axis represents the angle in degree. Furthermore, the reference input signal is square with frequency $0.0125 \mathrm{~Hz}$.

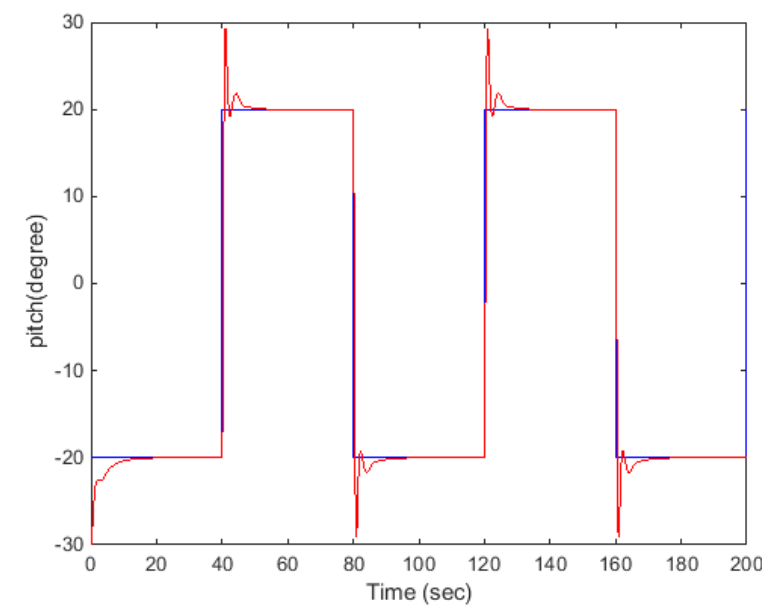

Fig.10. The results of the FF+LQR+I control of the pitch angle

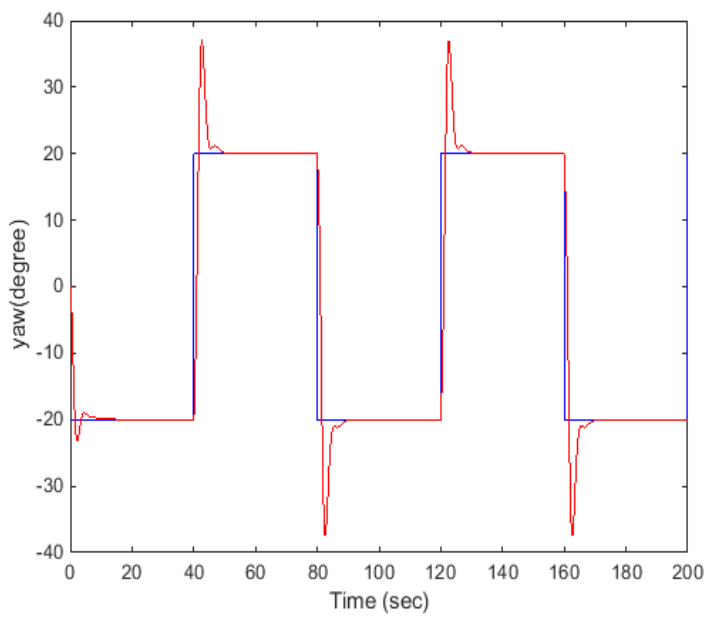

Fig.11. The results of the FF+LQR+I control of the yaw angle

The results reveal that the pitch response to the desired input is good with the aid of the controller. It follows the desired angle which is $20^{\circ}$ degrees, while there are oscillation and overshoot from $20^{\circ}$ to $29^{\circ}$ in about 5 seconds which led to producing $9^{\circ}$ oscillation above the $20^{\circ}$.

The overshoot can be approximately calculated from the curve by using the ratio of the peak value of the angle subtracted from the steady state over the steady state. Therefore, the pitch had $[(29-20) / 20] \times 100 \%=45 \%$ overshoot for about 5 seconds, then, settled down at $20^{\circ}$. The response of the yaw angle is similar to the pitch, but it had higher overshoot which arrived at $38^{\circ}$. Its oscillation was above the $20^{\circ}$ by $18^{\circ}$. It had $90 \%$ of overshoot in about 10 seconds for each square pulse.

PID controller was integrated to address the limitation in the original one (FF+LQR+I). Fig. 12 and Fig. 13 illustrate the results of the pitch and yaw angles after integrating this controller. The results were taken at $0.0125 \mathrm{~Hz}$ for the square reference input. 


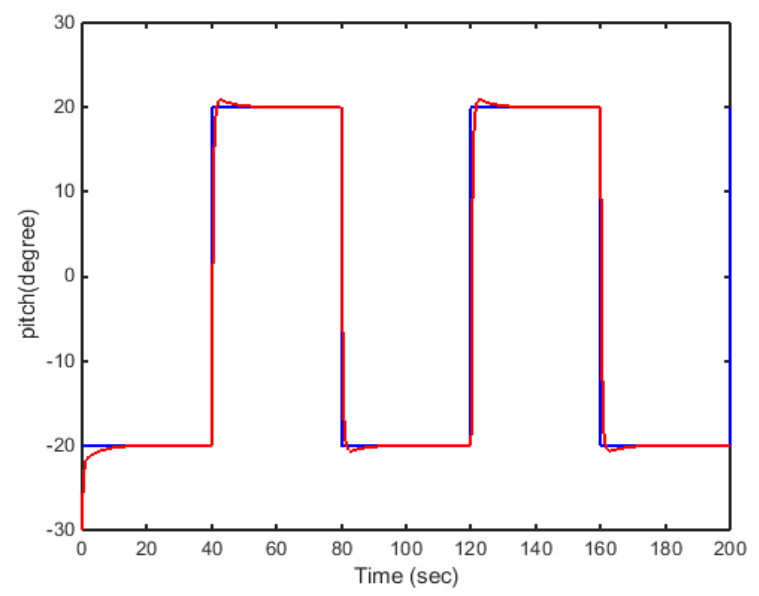

Fig.12. The results of the PID controller of the pitch angle

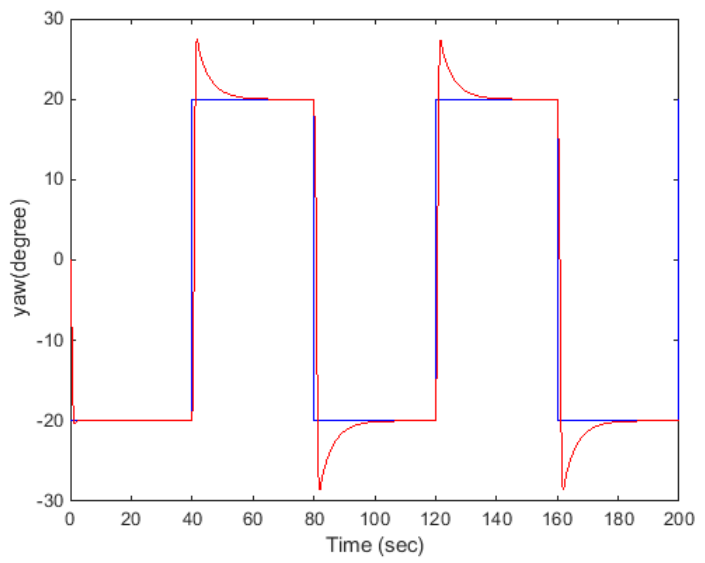

Fig.13. The results of the PID controller of the yaw angle

Fig. 13 shows that the yaw responded to the reference with an oscillation value equal to $28^{\circ}$ which is above the steady state by $8^{\circ}$. It overshot to $28^{\circ}$ for about 15 seconds and settled down to the steady state at $20^{\circ}$. The results of the FLC controller are shown in Fig. 14, Fig. 15, Fig. 16 and Fig. 17 respectively. The y-axis refers to the angle in degree, except for Fig. 14 where the y-axis refers to the angle in radian. It is presented in radian as this unit is clearer. The pitch tracked the reference with approximately $0 \%$ overshoot and low MSE which indicates an excellent response to the desired angle (Fig. 14 and Fig. 15).

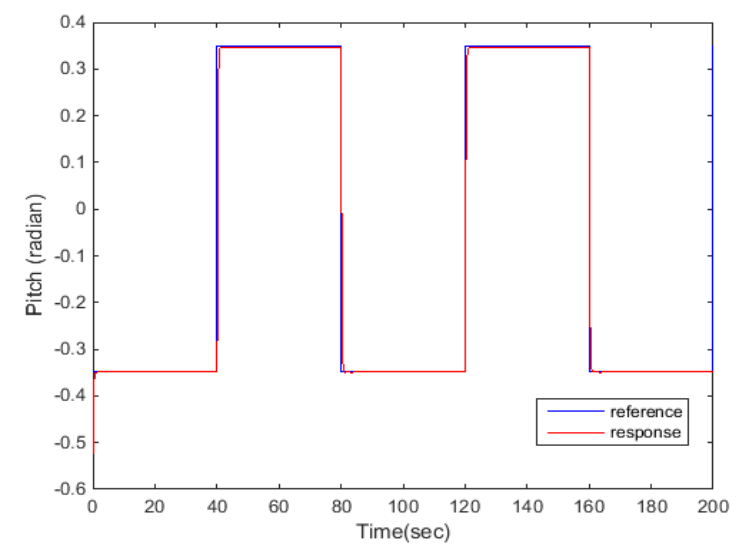

Fig.14. Simulation results of the pitch angle in radian under the fuzzy controller 


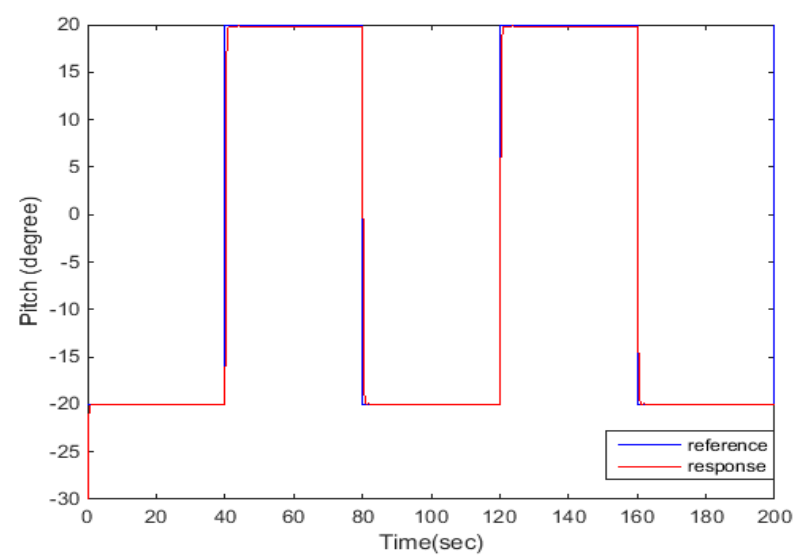

Fig.15. Simulation results of the pitch angle in degree under the fuzzy controller

The behaviour of the yaw, on the other hand, is more nonlinear than the pitch. Therefore, the FL parameters were adapted by using the ANFIS technique. It trained the data, reduced the error, and enhanced the response of the yaw. The ANFIS had for each square pulse $90 \%$ overshoot for about 5 seconds, and $50 \%$ undershoot for 15 seconds until it has settled down to the steady state. It overshot to $38^{\circ}$ for 5 seconds and damped to $10^{\circ}$ for 15 seconds. On the other hand, the negative part of the square wave had less percentage of overshoot (see Fig. 16).

More improvement was made by adapting the gain values of the yaw and making the pitch zero. This process assisted in reducing the MSE from 83.6618 (Fig. 16) to 38.119 (Fig.17). Fig. 17 shows that the yaw tracked the reference with less MSE in comparison to its outcomes before the improvement, while the yaw overshot to $50^{\circ}$ for the first 5 seconds and it had $50 \%$ undershoot for 5 seconds.

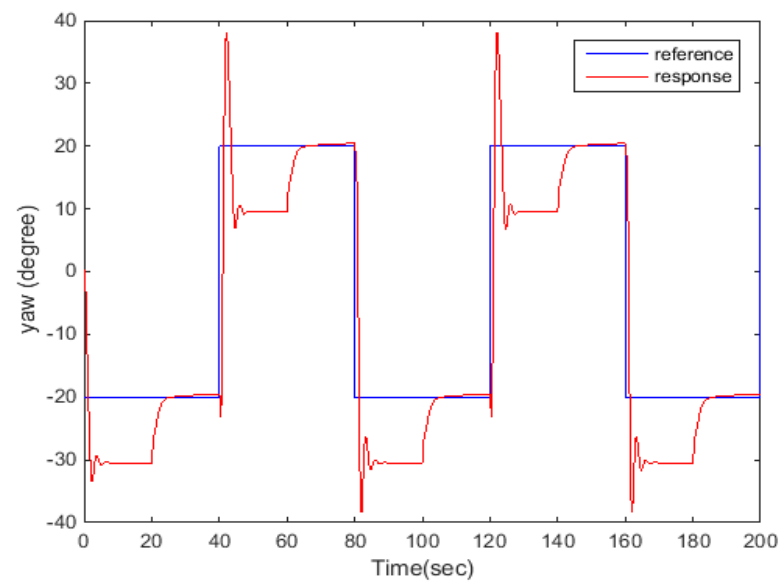

Fig.16. The results of the yaw angle in degree under FLC and ANFIS technique

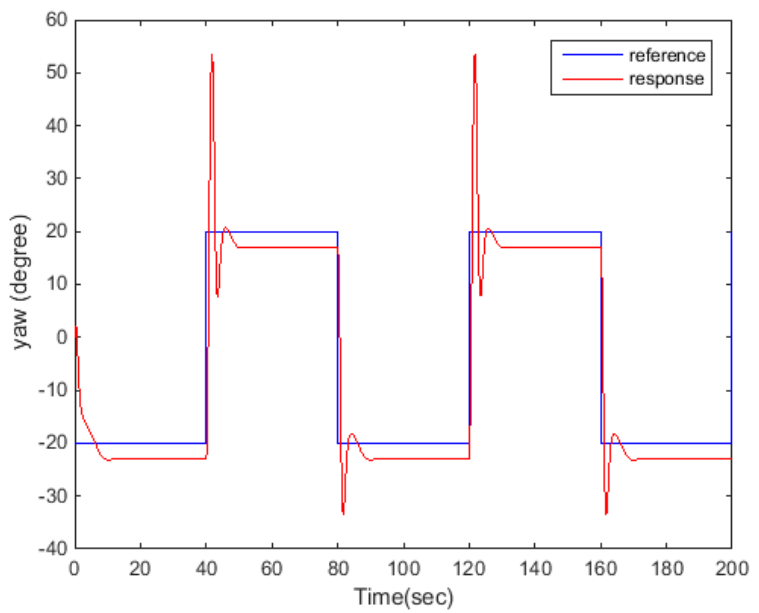

Fig.17. The results of the yaw angle in degree under FLC after adapting the gain values 
Moreover, the pitch under NARMA-L2 control tracked the reference, but it had a high overshoot in the first 5 seconds of each square pulse which reached to $100 \%$. Fig. 18 indicates that the pitch oscillated above the reference by $20^{\circ}$ for 5 seconds and settled down to the steady state. In the negative part of the square wave, it had a similar behaviour.

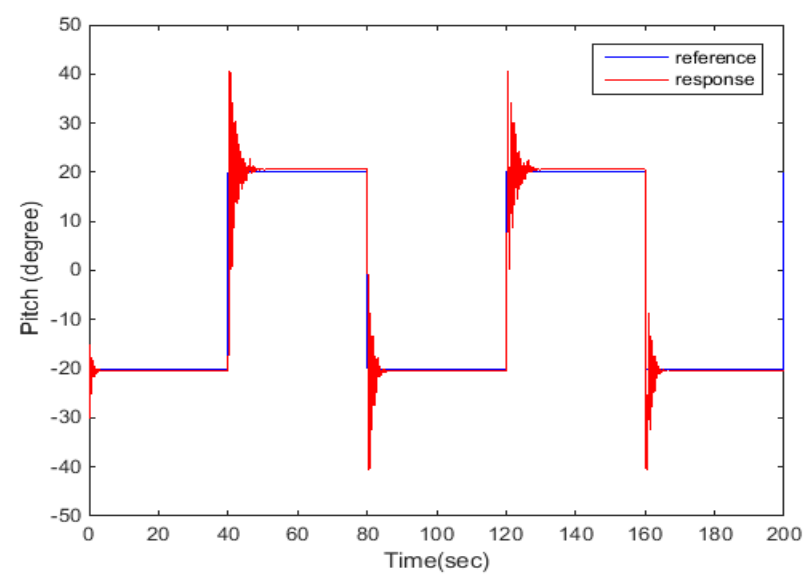

Fig.18. The results of the pitch angle with NARMA-L2

Fig. 19 shows the response of the yaw angle to the reference with NARMA-L2 control. It can be seen that the yaw failed to track the reference, and it oscillated under and above the reference with high MSE. Accordingly, it is required to create new random data that can make the output fits the response of the reference and follows it.

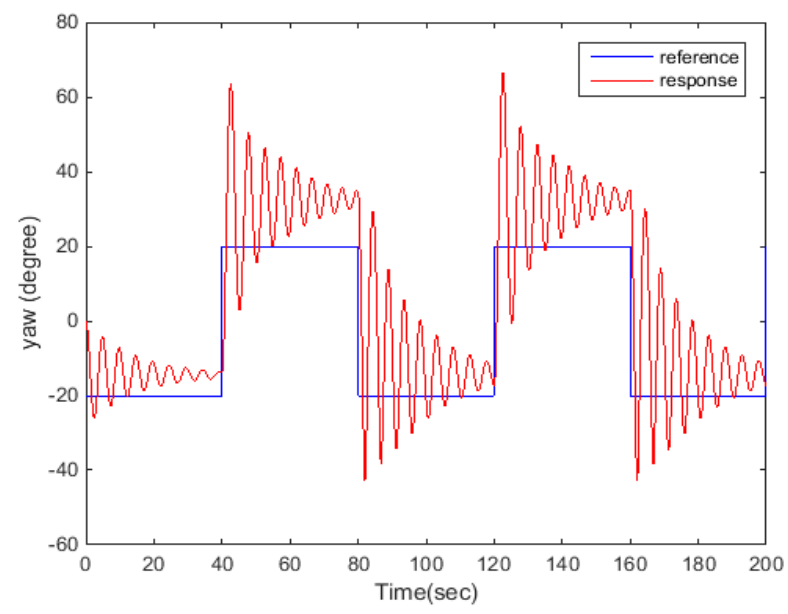

Fig.19. The results of the yaw angle with NARMA-L2

Tables 2 and 3 present the values of the MSE for both pitch and yaw with the original control, PID, FLC and NARMA-L2 controllers respectively. The values of the MSE for the pitch and yaw with the FLC are less than those with the NARMA-L2 controller, while the PID control has less MSE for the yaw than FLC. Both Tables also show the percentage of the overshoot which is better for the FL than NARMA-L2 and PID controllers for the pitch, whereas the PID control indicates less overshoot than the others for the yaw angle.

Table 2. The MSE and overshoot of the pitch

\begin{tabular}{|c|c|c|}
\hline Type of Control & Mean square error (MSE) & Overshoot (\%) \\
\hline LQR+FF+I & 9.2606 & $45 \%$ within the first 5 seconds \\
\hline PID & 7.6759 & $5 \%$ within the first 5 seconds \\
\hline Fuzzy Logic & 5.5367 & $0 \%$ \\
\hline NARMA-L2 & 11.4136 & $100 \%$ overshoot within the first 5 seconds \\
\hline
\end{tabular}


Table 3. The MSE and overshoot of the yaw

\begin{tabular}{|c|c|c|}
\hline Type of Control & Mean square error (MSE) & Overshoot (\%) \\
\hline LQR+FF+I & 24.5038 & $90 \%$ within the first 10 seconds \\
\hline PID & 17.1049 & $40 \%$ within the first 5 seconds \\
\hline Fuzzy Logic & 38.1199 & $150 \%$ overshoot within the first 5 seconds \\
\hline NARMA-L2 & 236.7748 & High overshoot for the whole signal \\
\hline
\end{tabular}

To sum up, FLC shows better control than the NARMA-L2 method and PID with less MSE for the pitch angle. However, the PID controller shows a better response to the yaw angle in comparison to the FLC and NARMA-L2 These findings are in agreement with the study conducted by Singh \& Panjwani [7] where it was indicated that the use of FL method had an improved effect on the nonlinear two links of a rigid manipulator.

\section{Conclusions}

It can be inferred that the use of FLC could provide a more accurate response, less MSE, and less overshoot in comparison to the application of the NARMA-L2 based on neural network controller. However, NARMA-L2 control result is good for the pitch angle and it may provide better results after performing an optimization process. In addition, the results of the PID control was the best one for the yaw angle.

Based on the overall findings, further research is required. Firstly, greater improvement for the yaw angle by using the FLC is needed to reduce the MSE as much as possible and increase its accuracy to that of the pitch angle. Secondly, optimization of the NARMA-L2 control of the yaw angle by using the swarm optimization may improve the response of the system under this control. Finally, it is also anticipated that the combination of the FLC for controlling the pitch angle and the PID may produce more accurate outcomes.

\section{References}

[1] M. Hernandez-Gonzalez, A. Y. Alanis, and E. A. Hernandez-Vargas, "Decentralized Discrete-time Neural Control for a Quanser 2-DOF Helicopter," Appl. Soft Comput., vol. 12, no. 8, pp. 2462-2469, 2012.

[2] D. Iakovou, "Fuzzy Control for Helicopter Aviation," Master thesis, University of Twente, 2002.

[3] G. Barbaroso, L. Ozdamar, and E. Ahmet, "An Interactive Approach for Hierarchical Analysis of Helicopter Logistics in Disaster Relief Operations,” Eur. J. Oper. Res., vol. 140, pp. 118-133, 2002.

[4] G.-R. Yu and H.-T. Liu, "Sliding Mode Control of a Two-degree-of-freedom Helicopter via Linear Quadratic Regulator," IEEE Int. Conf. Syst. Man Cybern., vol. 4, 2005.

[5] "PilotFriend," 2016. [Online]. Available: http://www.pilotfriend.com/training/flight_training/rotary/helis.htm. [Accessed: 03Aug-2016].

[6] B. Smaoui and M. Gabr, "The Box-Jenkins Analysis and Neural Networks: Prediction and Time Series Modelling," Applied Mathematical and Modelling," vol. 27, no. 10, pp. 805-815, 2003.

[7] N. Singh and B. Panjwani, "Comparison of Neural Network and Fuzzy Logic Control for Nonlinear Model of Two Link Rigid Manipulator," Int. J. Control Autom., vol. 7, no. 4, pp. 417-428, 2014.

[8] M. A. Mohd Basri, A. R. Husain, and K. A. Danapalasingam, "Intelligent Adaptive Backstepping Control for MIMO Uncertain Non-linear Quadrotor Helicopter Systems," Trans. Inst. Meas. Control, vol. 37, no. 3, pp. 345-361, 2015.

[9] F. Chetouane and S. Darenfed, "Neural Network NARMA Control of a Gyroscopic Inverted Pendulum," Eng. Lett., vol. 16, no. 3, pp. 274-279, 2008.

[10] A. Bousbaine, A. Bamgbose, G. Poyi, and A. K. Joseph, "Design of Self-tuning PID Controller Parameters Using Fuzzy Logic Controller for Quad-rotor Helicopter," Int. J. Trend Res. Dev., vol. 3, no. 6, pp. 95-99, 2016.

[11] R. Islam, K. Abdullah, N. Parveen, and M. Mahmud, "Adaptive Control of Nonlinear System Based on QFT Application to 3 DOF Flight Control System,” Telkomnik, vol. 17, no. 5, pp. 2595-2606, 2019.

[12] Quanser, "Position Control 2-DOF Helicopter Reference Manual."

[13] “Quanser 2-DOF Helicopter Laboratory Manual," 2011.

[14] F. A. Salem and A. A. Rashed, "PID Controllers and Algorithms: Selection and Design Techniques Applied in Mechatronics Systems Design - Part II," vol. 2, no. May, pp. 191-203, 2013.

[15] F. Dernoncourt, Introduction to Fuzzy Logic Control. Massachusetts Institute of Technology, available at: file:///C:/Users/CCC/Downloads/fuzzy_logic1.pdf MIT, pp. 1-19, 2013.

[16] A. Kusagur, S. F. Kodad, and B. V. S. Ram, "Modeling, Design and Simulation of an Adaptive Neuro-Fuzzy Inference System (ANFIS) for Speed Control of Induction Motor," Int. J. Comput. Appl., vol. 6, no. 12, pp. 29-44, 2010.

[17] M. T. Hagan, H. B. Demuth, and O. DE Jesus, "An Introduction to the Use of Neural Networks in Control Systems," Int. J. Robust Nonlinear Control, vol. 12, no. 11, pp. 959-985, 2002.

[18] C. Jeyachandran and M. Rajaram, "Neural Network Based Predictive, NARMA-L2 and Neuro-Fuzzy Control for a CSTR Process," J. Eng. Appl. Sci., vol. 5, no. 3, pp. 30-42, 2011.

[19] D. F. Ahmed and A. H. Khalaf, "Artificial Neural Networks Controller for Crude Oil Distillation Column of Baiji Refinery,” J. Chem. Eng. Process Technol., vol. 07, no. 01, pp. 1-9, 2016. 


\section{Authors' Profiles}

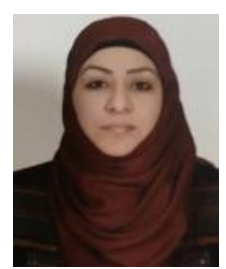

Noor Al-Fallooji was born in 1983. She received BSc Degree in Electrical Engineering from the University of Babylon in 2004, MSc in Advanced Electronic and Electrical Engineering from Brunel University London/ United Kingdom in 2016. Her research focuses on Artificial Intelligence and Power Distribution. She is currently an electrical engineer in the General Electricity Distribution Company in Iraq. Her main job is as a supervisor and executive engineer in many projects.

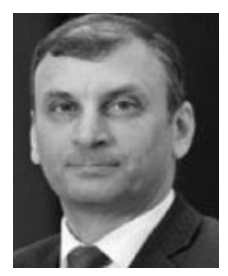

Maysam Abbod received the Ph.D. degree in control engineering from The University of Sheffield, U.K., in 1992. He is currently a Reader 919 of electronic systems with the Department of Electronic and Computer Engineering, Brunel University London, UK. He has authored more than 50 papers in journals, nine chapters in edited books and more than 50 papers in refereed conferences. His current research interests include 925 intelligent systems for modeling and optimization. He is a member of the IET, UK, and a Chartered Engineer in U.K. He is serving as an Associate Editor for the Engineering Application of Artificial Intelligence Elsevier.

How to cite this paper: Noor Salam Al-Fallooji, Maysam Abbod, "Helicopter Control Using Fuzzy Logic and Narma-L2 Techniques", International Journal of Intelligent Systems and Applications(IJISA), Vol.12, No.5, pp.1-14, 2020. DOI: 10.5815/ijisa.2020.05.01 